\title{
Diversity of Azoles Resistant Aspergillus Species Isolated from Experience and Naïve Soils in Nairobi County and Naivasha Sub-County Kenya
}

\author{
Edson K. Kemoi \\ Jomo Kenyatta University of Agriculture and Technology, Kenya \\ University of Kabianga, Kenya \\ Andrew Nyerere \\ Jomo Kenyatta University of Agriculture and Technology, Kenya \\ Uwe Gross \\ Oliver Bader \\ University Medical Center Gottingen, Germany \\ Tohru Gonoi \\ Chiba University \\ Christine C. Bii \\ Kenya Medical Research Institute, Kenya
}

Doi: 10.19044/esj.2017.v13n36p301 URL:http://dx.doi.org/10.19044/esj.2017.v13n36p301

Abstract

New triazole antifungals voriconazole, itraconazole and posaconazole are recommended for prophylaxis and treatment of both invasive and chronic fungal infections such as aspergillosis and aspergilloma. Emergence of azole-resistant among $A$. fumigatus isolates have been reported in other countries including Tanzania ascribed to either previous antifungal treatment, prophylaxis or triazoles use in agriculture. The use of azole based fungicides in the robust horticulture in Kenya is a significant risk factor for antifungal resistance. The study proposes to analyze environmental isolates of Aspergillus fumigatus, Aspergillus flavus and Aspergillus niger for the presence of resistance against the triazoles antifungals. Fungicide naïve soils were obtained from uncultivated virgin fields while fungicide experience soils were collected from flower, agricultural and horticultural fields and greenhouses within Naivasha sub-county and Nairobi County. The fungal isolates were subjected to antifungal susceptibility to triazoles using broth micro dilution method. A total of 492 samples were analyzed in Nairobi, 52 isolates were identified and they resistance were as follow: A. fumigatus (32\%), A. niger (26.09\%), A. flavus (33.33\%) and A.terreus (0\%) and in Naivasha 44 isolates were isolated out of which 25 were A. fumigatus and its 
resistance was at 36\%. Data were analyses using student $\mathrm{T}$ test and showed they no different between resistant and susceptible isolates from the two location. Data generated will serve to inform on the current status of triazoles resistance pattern and to raise concern emerging antifungal resistance in clinical practice.

Keywords: Azoles resistant Aspergillus, Environmental isolates, Aspergillus fumigatus

\section{Introduction}

Aspergillus species are found worldwide, it has isolated in organic matter, soil and decaying organic matter, Most of the Aspergillus species sporulated highly (Balajee, 2009, Mann et al., 2003). The conidia hence is carried by wind and inhaled causing infection in individual whose immune system is compromised (Perfect et al., 2001). Azoles namely posaconazole, Voriconazole and intraconazole are drugs used as first line in treatment of aspergillosis (Walsh et al., 2008). Other drugs with sensitive against Aspergillus species are Anidulafungin, micafungin, caspofungin and amphotericin B. Aspergillus fumigatus which causes most of the chronic and invasive aspergillosis (Denning et al., 2003, Walsh et al., 2008) is normally sensitive to the three classes of antigungal. However the recent past cases of patient with aspergillosis caused by A. fumigatus resistant to azoles has been reported (33). Aspergillus terreus is resistant to amphotericin B while Aspergillus pseudofischeri and A. lentulus is resistant to azoles intrinsically (Alcazar et al., 2008).

Azole resistance in Aspergillus infection due to azole treatment failure is an emerging health problem (Van der Linden et al., 2013. Van der Linden et al., 2011, Vermeulen et al., 2013). Surveillance reports have showed geographical spread of azole resistant species in clinical and environment in Africa, Europe, Asia, North and South America and Middle East (Vermeulen et al., 2013, Chowdhary et al., 2014). In contrast Netherland surveillance studies showed that azole resistance is endemic, TR46/Y121F/T289A and TR34/L98H cyp51A gene mediated resistance been the most common (Van der Linden et al., 2011).Multicenter international surveillance network study reported $3.2 \%$ triazoles resistance. Majority of resistant isolates were Aspergillus species at $78 \%$ and $22 \%$ of the other sibling species (A. lentulus, A. udagawae and A. thermomutatus) (Lamoth, 2016, Van der Linden et al., 2015).

The common reported mechanism of azoles resistance is alteration of the azoles drugs target i.e. lanosterol 14- $\alpha$-demethylase and mutation in gene cyp51A (Chen et al., 2005, Mellado et al., 2005, Mellado et al., 2004, DiazGuerra et al., 2003, Mann et al., 2003). Substitution usually occurs in codon 
98 by usually by substitution of histidine with leucine usually in the promoter region i.e. TR34/L98H. Some authors have attribute widespread uses of azoles in agricultural may have led to selection of strain carrying the resistant gene (Snelders et al., 2012, Verweiji et al., 2009). The use of azole based fungicides in the robust horticulture in Kenya is a significant risk factor for antifungal resistance. Recently several authors have reported a novel CYP51A mediated resistance with high resistance level to Voriconazole, TR46/Y121F/T289A also has be isolated from clinical sources and environmental in Europe, Africa, Latin America and Indian (Verweij et al., 2015,Abdolrasouli et al., 2016, Le Pape et al., 2016, Fuhren et al., 2015). The aims of this study were to investigate the diversity and prevalence of azoles resistant Aspergillus species in Nairobi County and Naivasha sub-County.

\section{Materials and methods}

\section{c. Sampling for environmental isolates}

Approximately $5 \mathrm{~g}$ dry top surface soils from agricultural site were collected into a sterile $15 \mathrm{ml}$ Falcon tube using a sterile plastic spoon. Soil samples were transported in a leak proof packaging in a cool box to KEMRI-Center for Microbiology Research for mycological investigations.

d.

e. Fungal culture and identification

Approximately a gram of the soil sample were suspended and vortexed thoroughly in $5 \mathrm{ml}(0.5 \% \mathrm{w} / \mathrm{v})$ saponin. The debris allowed to settle and the supernatant transferred to a fresh tube. The resulting suspension were then centrifuged, and the pellet suspended in a final volume of $500 \mu$ l sterile $\mathrm{NaCl}$. Hundred $\mu$ icrolitres of the suspension were plated on Sabouraud dextrose agar containing; (a) no drug, (b) $1 \mu \mathrm{g} / \mathrm{ml}$ Itraconazole (c) $1 \mu \mathrm{g} / \mathrm{ml}$ Voriconazole. All the Plates were incubated $30^{\circ} \mathrm{C}$ for 72 hours, checking daily for any growth. Colonies growing after incubation on triazoles free media were used as control and to access fungal diversity, while triazoles containing agar were used to determine resistant isolates.

\section{Triazoles susceptibility testing}

Minimum inhibitory concentrations to Itraconazole (ITZ), Voriconazole (VCZ) and Posaconazole (PSZ) were tested by broth micro dilution according to the EUCAST reference method (EUCAST, 2008) with minor modification.

Exactly $9.6 \mathrm{mg}$ of azoles (Itraconazole, Voriconazole, posaconazole) powder were weighed and dissolved in $3.0 \mathrm{ml}$ of DMSO to get $1600 \mu \mathrm{g} / \mathrm{ml}$ stock. Ten sterile tubes were used for serial dilution in each tube $3 \mathrm{mls}$ of DMSO were added. Starting with the first tube $3 \mathrm{mls}$ of the stock solution 
were added, mix and 3mls form the mixed of the first tube was transfer to second tube mixed and pipette $3 \mathrm{mls}$ from the second and transfer to the third tubes and the process were continued up to the ten tubes. The second serial dilution were done using ten tubes, in each of the tubes (From first serial dilution tubes) $4.9 \mathrm{ml}$ of RPMI were added to each of the ten tubes

From each tubes of the second dilution $200 \mu 1$ were dispensed to microtitre plates and inoculated with $10 \mu \mathrm{l}$ of the inoculums. Plates were then incubated at $30^{\circ} \mathrm{C}$ for $72 \mathrm{~h}$. The MIC values of all drugs were determined visually as the lowest concentrations with no visible growth. EUCAST drug susceptible controls strains DSM819 and ATCC46645 were used while azole resistant control isolates allele TR/L98H) and (allele G54W, ITZR + PSZR86) will be used.

\section{Results}

A total of 492 of both Naïve and experience soils (246 Naïve soils and 246 experienced soils) were analyzed. At total 52 isolates were isolated from experience soil in Nairobi out which proportions of azoles resistance were as follows: A. fumigatus (32\%), A. niger (26.09\%), A. flavus $(33.33 \%)$ and $A$.terreus $(0 \%)$ as showed in table I. In Naivasha a total of 44 isolates were isolated, out of which 25 A. fumigatus were isolated and $36 \%$ were azole resistant as showed in Table 1 .

Table 1: Showing isolates from experience soils both in Nairobi and Naivasha

\begin{tabular}{|c|c|c|c|c|c|c|}
\hline \multicolumn{4}{|c|}{ Nairobi Experience soils isolates } & \multicolumn{3}{c|}{ Naivasha experienced soils } \\
Isolates
\end{tabular}

Out of 246 naïve soils analyzed In Nairobi a total of 40 isolates were isolated, of which resistance were as follows: A. fumigatus (19.23\%), A niger (23.08\%), A. flavus (0\%), and A. terreus (0\%) as showed in table 2. In Naivasha 50 Aspergillus species were isolated of which resistance were as follows: A. fumigatus (19.05\%), A niger (15.38\%), A. flavus (0\%), and $A$. terreus $(0 \%)$ as showed in the table 2 below.

Table 2: Showing isolates from Naïve soils both in Nairobi and Naivasha

\begin{tabular}{|c|c|c|c|c|c|c|}
\hline \multicolumn{4}{|c|}{ Nairobi Naïve soils isolates } & \multicolumn{3}{c|}{ Naivasha Naïve soils Isolates } \\
\hline $\begin{array}{c}\text { Total } \\
\text { no. }\end{array}$ & Susceptible & Resistant's & $\begin{array}{c}\text { Total } \\
\text { no. }\end{array}$ & Susceptible & Resistant's \\
\hline fumigatus & 26 & $21(80.77 \%)$ & $5(19.23 \%)$ & 21 & $17(80.95 \%)$ & $4(19.05 \%)$ \\
\hline niger & 13 & $10(76.92 \%)$ & $3(23.08 \%)$ & 26 & $22(84.62 \%)$ & $4(15.38 \%)$ \\
\hline
\end{tabular}




\begin{tabular}{|c|c|c|c|c|c|c|}
\hline flavus & 0 & $0(0.0 \%)$ & $0(0.0 \%)$ & 3 & $3(100 \%)$ & $0(0.0 \%)$ \\
\hline terreus & 1 & $1(100 \%)$ & $0(0.0 \%)$ & $0(0.0 \%)$ & $0(0.0 \%)$ & $0(0.0 \%)$ \\
\hline Total & 40 & $32(80.0 \%)$ & $8(20.0 \%)$ & 50 & $42(84.0 \%)$ & $8(16.0 \%)$ \\
\hline
\end{tabular}

T-test comparison of the resistant samples of Nairobi Naïve soils isolates to the Nairobi Experience soils isolates

The data was first normalized by getting the percentage resistance of the fungi. These were therefore used in testing for the variations. Using twosample t-test, there was no significant difference in percentage resistant isolates in Nairobi naïve samples (mean 6.2\%) to the percentage resistance isolates in Nairobi experience isolates (mean 7.8\%), $(\mathrm{T}=1.24, \mathrm{P}=0.271)$.

\section{Two-Sample T-Test and CI: Nairobi Naive, Nairobi experience}

Two-sample T for Nairobi Naive soils vs Nairobi experience soils

$\mathrm{N}$ Mean StDev SE Mean

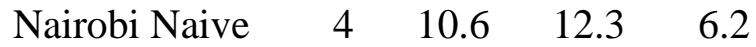

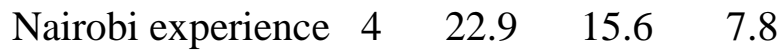

Difference $=$ mu N. Naive - mu N. experience

Estimate for difference: -12.28

95\% CI for difference: $(-37.78,13.23)$

$\mathrm{T}$-Test of difference $=0($ vs not $=): \mathrm{T}-$ Value $=-1.24 \mathrm{P}-$ Value $=0.271 \mathrm{DF}=5$

T-test comparison of the resistant samples of Nairobi Naïve soils isolates to the Naivasha naïve soil isolates

The result showed that there was no significant difference in percentage resistant isolates in Nairobi naïve samples (mean 6.2\%) to the percentage resistant isolates in Naivasha naive isolates (mean 5.0\%), $(\mathrm{T}=$ $0.25, \mathrm{P}=0.814$ ).

Two-Sample T-Test and CI: Nairobi Naive, Naivasha naive

Two-sample T for Nairobi Naive soils vs Naivasha naïve soils

N Mean StDev SE Mean

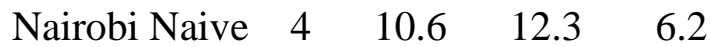

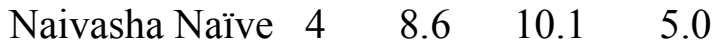

Difference $=$ mu N. Naive - mu Naivasha naive

Estimate for difference: 1.97

95\% CI for difference: $(-18.46,22.40)$

$\mathrm{T}$-Test of difference $=0(\mathrm{vs}$ not $=)$ : T-Value $=0.25 \mathrm{P}-$ Value $=0.814 \mathrm{DF}=5$ 
T-test comparison of the resistant samples of Naivasha Naïve soil isolates to the Naivasha experience soil isolates

The result showed that there was no significant difference in percentage resistant isolates in Naivasha naïve samples (mean 5.0\%) to the percentage resistant isolates in Naivasha experience isolates (mean 8.2\%), $(\mathrm{T}=1.51, \mathrm{P}=0.206)$.

Two-Sample T-Test and CI: Naivasha experience, Naivasha naive

Two-sample $\mathrm{T}$ for Naivasha experience vs Naivasha naive

$\mathrm{N}$ Mean StDev SE Mean

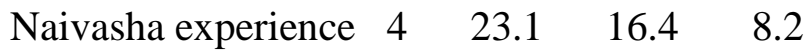

$\begin{array}{lllll}\text { Naivasha naive } & 4 & 8.6 & 10.1 & 5.0\end{array}$

Difference $=$ mu Naivasha experience - mu Naivasha naive

Estimate for difference: 14.50

95\% CI for difference: $(-12.18,41.17)$

$\mathrm{T}$-Test of difference $=0($ vs not $=): \mathrm{T}$-Value $=1.51 \mathrm{P}-$ Value $=0.206 \mathrm{DF}=4$

T-test comparison of the resistant samples of Naivasha Naïve soil isolates to the Nairobi experience soil isolates

The result showed that there was no significant difference in percentage resistant isolates in Naivasha naïve samples (mean 5.0\%) to the percentage resistant isolates in Nairobi experience isolates (mean 7.8\%), (T $=1.54, \mathrm{P}=0.185)$.

Two-Sample T-Test and CI: Nairobi experience, Naivasha naive

Two-sample T for $\mathrm{N}$. experience vs Naivasha naive

N Mean St Dev SE Mean

$\begin{array}{lllll}\text { Nairobi experience } & 4 & 22.9 & 15.6 & 7.8\end{array}$

$\begin{array}{lllll}\text { Naivasha Naïve } \quad 4 & 8.6 & 10.1 & 5.0\end{array}$

Difference $=$ mu Nairobi experience $-\mathrm{mu}$ Naivasha naive

Estimate for difference: 14.25

95\% CI for difference: $(-9.56,38.06)$

$\mathrm{T}$-Test of difference $=0($ vs not $=): \mathrm{T}-$ Value $=1.54 \mathrm{P}-$ Value $=0.185 \mathrm{DF}=5$

\section{Discussion}

We report the detection of environmental azole resistance Aspergillus species from both Naïve and experience soil in Nairobi County and Naivasha sub-County, Kenya. In experience soils, there were high percentages in resistance from Naivasha isolates this may be due to irrational used of 
fungicides in large scale horticultural farming as compare to Nairobi which was mostly small-scale farming

In the recent decades, there has an increase in opportunities infection one of them is aspergillosis (Walsh et al., 2008). Invasive fungal infection diseases are associated by high mortality and morbidity, partially because of failure to diagnosis early resulting in delay treatment. Most of the cross resistance in Aspergillus species by several authors have be reported infrequently, which mean they are infrequent to date. Low prevalence is due to variation in laboratory testing between different laboratories.

The detection of azole resistance of Aspergillus species was done based on medium containing azoles and micro dilution susceptibility testing. The uses of azole containing in screening environmental samples for the presence of resistance were first described using Itraconazole at a concentration of $4 \mathrm{mg} / \mathrm{liter}$ (Snelders et al., 2009). Since then breakpoint have been proposed to be $1 \mathrm{mg} /$ liter for Itraconazole (Pfaller et al., 2009, Verweji et al., 2009). Which raises question that resistance isolates may go undetected. However it has been reported by several authors the MIC of most isolates against Itraconazole is $8 \mathrm{mg} / \mathrm{liter}$ or more (Howard et al., 2009,). In the study we further included Voriconazole (1mg/liter) and posaconazole (1mg/liter) SDA agar. Student T test showed that they were no significant difference in percentage resistance $(\mathrm{P}=0.271)$ of isolates from Nairobi naïve and experience soils isolates as well as in Naivasha from naïve and experience soils $(\mathrm{P}=0.206)$.

The used of azole containing agar, hypothetically may results in laboratory generation of resistance in the recover isolates though expose. However it is believe that since de novo short azole exposure (Almost $72 \mathrm{hr}$ exposure) is for short time it may not cause resistance and detection of cyp51A alterations which cause necessary (Mellado et al., 2007). Acquire azole resistance usually develops due to response of fungi to azole exposure in patients or in agricultural (Chen et al., 2005, Howard et al., 2006, Verweij et al., 2009). Drug expose over a long periods and high numbers of reproducing fungi are some of the factors that played a role in the selection of resistance (Anderson, 2005). This condition is met in soil when agricultural azole applied remained in soils over a year and in patients with Cavitary Aspergillus (Hof, 2001).

Demethylation inhibitors and Azoles fungicides are recommend for used in plant protection (Hof, 2001). In Netherlands the used for fungicides in agricultural has double since mid1990s (Verweji et al., 2009). Similar trends has been observed in Denmark. Resistant Aspergillus fumigatus is selected by used of azole fungicides or it can be introduced via importation of commercial compost to the environment, are one of the key factors in development of resistance (Verweji et al., 2009). In our study most of the 
sites with resistant Aspergillus were previously exposed to azoles fungicides before. This is always hard to management aspergillosis because most laboratories do not perform sensitivity test and due to negative cultures. Hence the prevalence of azoles resistant Aspergillus species may be underestimated with risk of inappropriate therapy. We believe in our study and emerging reports on azole related resistance, we suggest that susceptibility testing for Aspergillus species should be performed routinely.

\section{Conclusion}

The study found out that they are triazoles resistance Aspergillus species in the two county. High resistance in Naivasha sub county soils show that resistance could be catalyst by application of fungicides in agriculture. This posed a serious health public concern and more surveillance should done in the country.

Funding: This study was funded by Kenya Medical Research Institute (KEMRI IRG Grant no. (INNOV/IGR/019/2).

Conflict of Interest: The authors declare that they have no conflict of interest.

Ethical approval: The study was approved by Kenya Medical Research Institute, Scientific and ethics Review Unit (KEMRI/SERU/P000602/3031).

Informed consent: There were no human participation or direct human contact. However Approved permission to access the farms were obtained from relevant institution or flower farms owner or individual farm owner.

\section{References:}

1. Alcazar-Fuoli L., Mellado E., Alastruey-Izquierdo A., CuencaEstrella M., Rodriguezx-Tudela L.j. (2008). Aspergillus section fumigati: Antifugal susceptibility patterns and sequence-based identification. Antimicrob. Agents. Chemother. 52:1244-1251.

2. Balajee S.A. (2009). Aspergillus terreus complex. Med. Mycol. 47: S42-S46.

3. Denning D.W., Riniotis K., Dobrashian R., Sambatakou H. (2003). Chronic cavitary and fibrosing pulmonary and pleural aspergillosis: case series, proposed nomenclature change and review. Clin. Infect. Dis. 37:S265-S280.

4. Mann P.A., Parmegianio R.M., Wei S.Q., Mendrick C.A., Li X., Loebenberg D., Didomenico B., Hare R.S., Walker S.S., McNicholas P.M. (2003). Mutations in Aspergillus fumigatus resulting in reduced susceptibility to posaconazole appear to be restricted to a single amino acid in the cytochrome P450 14aplpha-demethylase. Antimicrob. Agents. Chemother. 47:577-581. 
5. Perfect J.R., Cox G.M., Lee J.Y., Kauffman C.A., Chapman S.W., Morrison V.A., Pappas P., Hiemenz J.W., Stevens D.A. (2001). The impact of culture isolation of Aspergillus species: a hospital-based survey of aspergillosis. Clin. Infect. Dis. 33:1824-1833.

6. Walsh T.J., Anaissie E.J., Denning D.W., Herbrecht R., Kontoyiannis D.P., Marr K.A., Morrison V.A., Segal B.H., Steinbach W.J., Stevens D.A., van Burik J.A., Wingard J.R., Patterson T.F. (2008). Treatment of aspergillosis: clinical practice guidelines of the infectious Diseases Society of America. Clin. Infect. Dis. 46:327-360.

7. Chen J. Li H. Li R. Bu D. Wan Z. (2005). Mutations in the cyp51A gene and susceptibility to Itraconazole in Aspergillus fumigatus serially isolated from a patient with lung aspergilloma. J. Antimicrob. Chemother. 55:31-37.

8. Diaz-Guerra T.M. Mellado E. Cuenca-Estrella M. Rodriguez-Tudela J.L (2003). A point mutation in the 14alpha-sterol demethylase gene cyp51A contributes to Itraconazole resistance in Aspergillus fumigatus. Antimicrob. Agents. Chemother. 47:1120-1124.

9. Mann P.A. Parmegiani R.M, Wei S.Q. Mendrick C.A. Li X. Loebenber D. Didomenico B. Hare R.S. Walker S.S. McNicholas P.M. (2003). Mutations in Aspergillus fumigatus resulting in reduced susceptibility to posaconazole appear to be restricted to a single amino acid in the cytochrome p450 14alpha-demethylase. Antimicrob. Agents. Chemother. 47: 577-581.

10. Mellado E. Garcia-Effron G. Alcazar-Fuoli L. Cuenca-Estrella M. Rodriguez-Tudela J.L. (2004). Substitutions at methionine 220 in the 14alpha-sterol demethlase (cyp51A) of Aspergillus fumigatus are responsible for resistance in vitro to azole antifungal drugs. Antimicrob. Agents Chemother. 48:2747-2750

11. Mellado E. Garcia-Effron G. Buitrago M.J.Alcarz-Fuoli L. CuencaEstrella M. Rodriguez-Tudela J.L. (2005). Targeted gene disruption of the 14-alpha sterol demethylase (cyp51A) in Aspergillus fumigatus and its role in azole drug susceptibility. Antimicrob. Agents Chemother. 49:2536-2538.

12. Verweij P.E. Snelders E. Kema D.H. Mellado E. Melchers W.J. (2009). Azole resistance in Aspergillus fumigatus: a side-effect of environmental fungicide use? Lancet infect. Dis. 9:789-795.

13. Anderson J.B. (2005). Evolution of antifungal-drug resistance: mechanism and pathogen fitness. Nat. Rev. Microbiol. 3:547-556.

14. Chen J. Li H. Li R. Bu D. Wan Z. (2005). Mutations in the cyp51A gene and susceptibility to Itraconazole in Aspergillus fumigatus serially isolate from a patient with lung aspergilloma. J. Antimicrob. Chemother. 55:31-37. 
15. Hof H. (2001). Critical annotations to the use of azole antifungals for plant protection. Antimicrob. Agents Chemother. 45:2987-2990.

16. Howard S.J. Webster I. Moore C.B. Gardiner R.E. Park S. Perlin D.S. Denning D.W. (2006). Multi-azole resistance in Aspergillus fumigatus. Int. J. Antimicrob. Agents 28:450-453.

17. Mellado E. Garcia-Effron G. Alcazar-Fuoli L. Melchers W.J. Verweij P.E. Cuenca-Estrella M. Rodriguez-Tudela J.1. (2007). A new Aspergillus fumigatus resistance mechanism conferring in vitro crossresistance to azole antifungals involves a combination of cyp51A alterations. Antimicrob. Agents Chemother. 51:1897-1904.

18. Howard S.J. Cerar D. Anderson M.J. Albarrag A. Fisher M.C. Pasqualotto A.C. Laverdiere M. Arendrup M.C. Perlin D.S. Denning D.W. (2009). Frequency and evolution of azole resistance in Aspergillus fumigatus associated with treatment failure. Emerg. Infect. Dis. 15:1068-1076.

19. Pfaller M.A. Diekema D.J. Ghannoum M.A. Rex J.H. Alexander B.D. Andes D. Brown S.D. Chaturvedi V. Espinel-Ingroff A. Fowler C.L. Johnson E.M. Knapp C.C. Motyl M.R. Ostrosky-Zeichner, Sheehan D.J. Walsh T.J. (2009). Wild-type MIC distribution and epidemiological cutoff standards institute broth micro dilution methods. J. Clin. Microbiol. 47:3142-3146.

20. Snelders E. Huis In't Veld A. Rijis A.J. Kema G.H. Melchers W.J. Verweiji P.E. (2009). Possible environmental origin of resistance of Aspergillus fumigatus to medical triazoles. Appl. Environn. Microbiol. 75:4053-4057.

21. Verweiji P.E. Howard S.J. Melchers W.J. Denning D.W. (2009). Azole-resistance in Aspergillus: proposed nomenclature and breakpoints. Drug Resist. Updat. 12:141-147.

22. Snelders E, Camps S.M. Karawajczyk A. Schaftenaar G. Kema G.H. Van der Lee H.A. Klaassen C.H. Melchers W.J. Verweij P.E.(2012). Triazole fungicides can induce cross-resistance to medical triazoles in Aspergillus Fumigatus. PloS one 7:e31801.doi:10.1371/journal.pone.0031801.

23. Verweij P.E. Snelders E. Kema G.H. Mellado E. Melchers W.J. (2009). Azole resistance in Aspergillus fumigatus: a side effect of environmental fungicide use? Lancet infect. Dis. 9:789-795.

24. Van der Linden J.W.M. Camps S.M.T. Kampinga G.A. Arends J.P.A. Debets-Ossenkopp Y.J. Haas P.J.A (2013). Aspergillosis due to Voriconazole highly resistant Aspergillus fumigatus and recovery of genetically related isolates from domiciles. Clin. Infect. Dis. 57:513-520. 
25. Van der Linden J.W.M. Sneldes E. Kampinga G.A. Rijnders B.J.A. Mattsson E. Debets-Ossenkopp Y.L (2011). Clinical implication of azole resistance in Aspergillus fumigatus, The Netherlands 20072009. Emerg. Infect. Dis. 17:1846-1854.

26. Vermeulen E. Lagrou K. Verweij P.E. (2013). Azoles resistance in Aspergillus fumigatus: a growing public health concern. Curr. Opin. Infect. Dis. 26-493-500.

27. Chowdhary A. Sharma C. Van den Boom M. Yntema J.B. Hagen F. Verweij P.E. (2014). Muitiazole resistant Aspergillus fumigatus in the environment in Tanzania. J. Antimicrob. Chemother. 69:2979-2983.

28. Lamoth F. (2016). Aspergillus fumigatus-related species in clinical practice. Front Microbiol. 7: 683.

29. Van der Linden J.W. Arendrup M.C. Warris A. Lagrou K. Pelloux H. Hauser P.M. Chryssanthou E. Mellado E. Kidd S.E. Tortorano A.M. (2015). Prospective multicenter international surveillance of azole resistance in Aspergillus fumigatus. Emerg. Infect. Dis. 21:10411044.

30. Verweij P.E. Ananda-Rajah M. Andes D,. Arendrup M.C. Bruggemann R.J. Chowdhary A. Cornely O.A. Denning D.W. Groll A.H. Izumikawa K. (2015). International expert opinion on the management of infection caused by azole-resistant Aspergillus fumigatus. Drug Resist. Updat. 21-22.

31. Abdolrasouli A. Rhodes J. Beale M.A. Hagen F. Rogers T.R. Chowdhary A. Meis J.F. Armstrong-James D. Fisher M.C. (2015). Genomic context of azole resistance mutations in Aspergillus fumigatus determined using whole-genome sequencing. MBio. 6:e00536.

32. Le pape P. Laverne R.A. Morio F. Alvarez-Moreno C. (2016). Multiple fungicide-drive alterations in azole-resistant Aspergillus fumigatus, Colombia. Emerg. Infect. Dis. 22:156-157.

33. Fuhren J. Voskuil W.S. Boel C.H. Haas P.J. Hagen F. Meis J.F. Kusters J.G. (2015). High prevalence of azole resistance in Aspergillus fumigatus isolates from high-risk patients. J. Antimicrob. Chemother. 70:2894-2898. 